\title{
THE FLOW OF WATER AND AIR THROUGH SOILS.
}

\author{
By J. WALTER LEATHER, Ph.D., F.I.O. \\ Agricultural Research Institute, Pusa, Bengul.
}

IN Part I of the current volume of this Journal Green and Ampt make certain assumptions regarding the factors which control the rate of flow of water and air through soils, the basis of which is that the channels in a soil may be regarded as a bundle of capillary tubes, and that the velocity of flow of a liquid will consequently conform to Poiseuille's formula. Such an assumption cannot however be maintained. Poiseuille's formula was deduced from the ascertained rate of flow of liquids through straight tubes of approximately uniform diameter; the latter being $\mathbf{1} \mathbf{~ m m}$. or so. The formula is not however really accurate even under these limited conditions, as was shown by Thorpe and Roger', who found it necessary to add the correction $-\rho \nabla / 8 \pi l t$. But the passages through the soil are not straight or of uniform diameter for any measurable distance, in fact they are wholly irregular, which Green and Ampt recognise, for they describe them as "irregular in area, length, direction and shape." But this being the case, Poiseuille's formula obviously cannot be expected to apply.

It is of course true that, having a certain cylinder full of soil, thoroughly saturated with water and through which water is flowing, the channels may assume fixed shape, and the velocity of flow would accordingly become uniform. Moreover, it is not difficult to fill a series of cylinders with portions of the same soil so nearly uniformly alike that the rate of flow of water through each will approximate to its neighbour. Therefore the agreement among the experimental results which Green and Ampt obtained with several portions of the same soil is not surprising. But this does not make their primary assumption legitimate. It is also to be noted that their experiments

\footnotetext{
1 Phil. Trans. 1894, A, Part II.
} 


\section{The Flow of Water and Air through Soils}

showed that the flow of water through any of the three directions adopted, did not in fact accord with the expectation which was arrived at from Poiseuille's formula.

The most interesting case which was tested is the flow through the horizontal column of soil from a water-reservoir. Here the acting force is simply the tension of the advanced water surface, and bere they found that the relation between the mass of water absorbed and the time, was a logarithmic one. Thus they arrive at a result similar to that which I deduced some years ago and dealt with in Memoir No. VI of the Chemical Series of the Imperial Department of Agriculture.

But as regards the downward movement of water through soils during wet weather, i.e. agricultural drainage, $I$ venture to refer to the explanation of its nature which has been published in Memoir No. X of the same series. The process cannot be likened to water flowing downwards through tubes. The advanced portion of the water spreads out in much the same manner as indicated by the curve, Fig. 3, in Green and Ampt's communication; the water forms a "wave," the "length" of which constantly increases whilst the "height" diminishes, unless the rainfall is very heavy and continuous. 